\title{
ON THE ADJOINT OF A CLOSED TRANSFORMATION ${ }^{1}$
}

\author{
A. BROWN
}

The main purpose of this note is to give a new proof of a known theorem. The basic "alternative" relations between an operator and its adjoint, of which Theorem 2 represents one possible formulation, are well known for bounded operators and offer no difficulty for closed operators if the spaces are reflexive. More recently the general case has been handled by several authors $[1 ; 3 ; 4 ; 5] .{ }^{2}$ The central idea of the present treatment is, by judicious normalization, to reduce Theorem 2 to Theorem 1, a result of some interest in its own right.

The following notation is employed: $E$ and $F$ are Banach spaces and $T$ is a closed linear transformation from $E$ to $F$ with dense domain $D ; E^{*}$ and $F^{*}$ are the dual spaces and $D^{*}$ is the domain of the adjoint transformation $T^{*}$; the ranges and null spaces of $T$ and $T^{*}$ are $R, R^{*}, N, N^{*}$ respectively.

It is well known (Closed Graph Theorem; see, e.g., [2, p. 41 and Theorem 2.12.3]) that $T$ is bounded if and only if $D=E$. However it seems to have been overlooked that the following straightforward dual assertion is also valid.

THEOREM 1. $T^{*}$ is bounded if and only if $D^{*}=F^{*}$.

Proof. One way is well known; if $T^{*}$ is everywhere defined, $T$ must be bounded (Banach-Steinhaus Theorem, see, e.g., [2, Theorem 2.12.4]) and then so is $T^{*}$. Suppose, on the other hand, that for some $M$ we have $\left\|T^{*} \phi\right\| \leqq M\|\phi\|$ for every $\phi \in E^{*}$. Let $\left\{\phi_{\nu}\right\}$ be a net in $D^{*}$ satisfying (i) $\left\|\phi_{\nu}\right\| \leqq 1$ for all $\nu$, and (ii) $\phi_{\nu} \rightarrow \phi_{0}$ in the weak* topology. The net $\left\{T^{*} \phi_{v}\right\}$ must converge to some element, say $f_{0}$, in the weak* topology. But then the net $\left\{\left(T^{*} \phi_{\nu}, \phi_{v}\right)\right\}$ converges weak* to $\left(f_{0}, \phi_{0}\right)$ in $E^{*} \oplus F^{*}=(E \oplus F)^{*}$ and, since the graph of $T^{*}$ is weak ${ }^{*}$ closed, we must have $f_{0}=T^{*} \phi_{0}$. In particular $\phi_{0} \in D^{*}$, so that the intersection of $D^{*}$ with the unit ball in $F^{*}$ is weak* closed. But then (see e.g., [2, Theorem 2.10.4]) $D^{*}$ is itself weak* closed and, since $D^{*}$ is weak* dense in $F^{*}$ in any case, the theorem is proved.

The following result corresponds with Lemma 324 of [4] and with Theorem 1.1 of [5] (see also [3]). It is also subsumed in the encyclopedic Theorem 1.1 of $[1]$.

TheOREM 2. (1) If $R$ is closed then $R^{*}=N^{0}$ (the annihilator of $N$ in

Received by the editors August 24, 1962 and, in revised form, January 28, 1963.

${ }^{1}$ Research on the note was supported, in the part, by the U. S. Air Force Office of Scientific Research.

2 The author is grateful to the referee for calling these references to his attention. 
$\left.E^{*}\right)$ so that $R^{*}$ is even weak* closed; moreover, $T^{*}$ is an open map of $D^{*}$ onto $N^{0}$.

(2) If $Q^{*}$ is (norm) closed then $R=N^{* 0}$ (the annihilator of $N^{*}$ in $F$ ) so that $R$ is also closed; moreover, $T$ is an open map of $D$ onto $N^{* 0}$.

Proof. Let $E_{1}=E / N$ and write $\pi$ for the natural projection, $\pi: E \rightarrow E_{1}$. Let also $F_{1}=\bar{R}$ and write $i: F_{1} \rightarrow F$ for the inclusion map. Finally, let $T_{1}$ denote the factorization of $T \bmod N$ so that $T=i \circ T_{1}$ $\circ \pi$. Then

$$
T^{*}=\pi^{*} \circ T_{1}^{*} \circ i^{*} \text {. }
$$

Indeed, if $T$ is bounded this is automatic; when $T$ is merely closed, the verification requires careful attention to the definition of $D^{*}$ but is still a routine chore which we omit. Moreover, it is easy to check that both $i^{*} \mid D^{*}$ and $\pi \mid D$ are open maps. It follows that there is no loss of generality in assuming that $T=T_{1}$, i.e., that $T$ is one-one and has dense range. In this special case the theorem reads:

(1') If $R=F$, then $T^{*}$ is an open map of $D^{*}$ onto $E^{*}$.

( $\left.2^{\prime}\right)$ If $\mathbb{R}^{*}$ is (norm) closed, then $T$ is an open map of $D$ onto $F$.

To complete the proof, we have but to invoke the fundamental relation

$$
\left(T^{-1}\right)^{*}=\left(T^{*}\right)^{-1}
$$

(Recall that the inverted graph of $-T^{*}$ is precisely the annihilator in $E^{*} \oplus F^{*}=(E \oplus F)^{*}$ of the graph of $T$.)

( $\left.1^{\prime}\right)$ If $R=F$, then $T^{-1}$ is everywhere defined and must be bounded. But then $\left(T^{-1}\right)^{*}$ is also everywhere defined and bounded, i.e., $T^{*}$ is open and onto.

$\left(2^{\prime}\right)$ If $R^{*}$ is norm closed it is complete and $\left(T^{*}\right)^{-1}$ must be bounded. But then by the preceding theorem $T^{-1}$ is bounded also, i.e., $T$ is open and onto.

COROLLARY. If the range of an adjoint operator is closed in the norm topology, it is automatically weak* closed.

\section{REFERENCES}

1. F. E. Browder, Functional analysis and partial differential equations. I, Math. Ann. 138 (1959), 55-79.

2. E. Hille and R. S. Phillips, Functional analysis and semigroups, Amer. Math. Soc. Colloq. Publ. Vol. 31, Amer. Math. Soc., Providence, R. I., 1957.

3. J. T. Joichi, On closed operators with closed range, Proc. Amer. Math. Soc. 11 (1960), 80-83.

4. T. Kato, Perturbation theory for nullity, deficiency and other quantities of linear operators, J. Analyse Math. 6 (1958), 261-322.

5. G.-C. Rota, Extension theory of differential operators. I, Comm. Pure Appl. Math. 11 (1958), 23-65.

RICE UNIVERSITY 